\title{
Laser Therapy in the Complex Prevention and Treatment of Covid-19 (Preliminary Results)
}

\section{V.A. Mikhaylov}

Eternity Medicine Institute, Dubai

*Corresponding Author: V.A. Mikhaylov, M.D. Ph.D., Kooperative str, 2/14, fl.96, 119048, Moscow, Russia, Eternity Medicine Institute, KG Tower, PO Box 120618, Dubai Marina, UAE.

Received date: December 15, 2020; Accepted date: January 04, 2021; Published date: January 08,2021

Citation: V.A. Mikhaylov. (2021) Laser Therapy in the Complex Prevention and Treatment of Covid-19 (Preliminary Results). International Journal of Clinical Case Reports and Reviews. 6(2); DOI:10.31579/2690-4861/101

Copyright: () 2021 V.A. Mikhaylov, This is an open-access article distributed under the terms of the Creative Commons Attribution License, which permits unrestricted use, distribution, and reproduction in any medium, provided the original author and source are credited.

\begin{abstract}
This article presents preliminary results of treatment of 51 patients with COVID 19 (Moscow Region, Russia). These patients were subjected to various schemes of immune stimulation for the prevention and treatment of this disease. Were comparedPercutaneus laser therapy (PLT), Intravenous Laser Blood Irradiation (ILBI), Drug stimulation and their combination. The results showed:

1. In the treatment of COVID-19, the use of various types of immunomodulation and anticoagulants proved to be most effective.

2. The combination of ILBI and TLT with immunomodulators proved to be the most effective in the prevention and treatment of COVID-19.

3. Immediate use of immunomodulators at the very beginning of COVID-19 reduces the severity of the disease, and facilitates its course.

Background and Aims: We started to use laser therapy in 1988. When used in different categories of cancer patients, it was found that various types of laser radiation stimulate the immune system. We started to use this peculiarity of laser therapy to boost the immune status of sickly patients with weakened immune systems, as well as for prevention and treatment of respiratory viral infections (e.g. influenza, parainfluenza, acute respiratory infections). We performed various types of Immunostimulation in 51 patients from Russia and evaluated its influence both on the morbidity and the course of COVID-19.
\end{abstract}

Rationale: Laser radiation $(890-910 \mathrm{~nm})$ stimulates cell immunity, increasing the amount of active T-lymphocytes. The wavelength of $630-640 \mathrm{~nm}$ is the most effective for irradiation both the blood and the vascular walls. At this wavelength photons are absorbed by oxygen, microcirculation improves, decrease blood viscosity, and direct impact on the nerve and muscle elements of the vascular wall influences the activity of the vascular and nervous systems.

Conclusion::The laser therapy practice we have been exercising for over 30 years has shown that it produces good immunostimulating effects. The use of various laser therapy methods combined with immunomodulatory drugs allow to reduce the number of patients infected with COVID-19, and reduce the severity of the disease.

Key words: immunostimulation; percutaneus laser therapy (plt); intravenous laser blood irradiation (ilbi); immunomodulatory drugs; covid-19; respiratory virus infection

\section{Introduction:}

In September 2019 we paid attention that there evolved an acute respiratory infection which had a clinical course different from regular flu clinic and the acute respiratory viral infections. The main manifestation of this infection were long-term and severe pneumonias, which did not respond well to the standard therapy. The computer tomography revealed interstitial edemas of the tissue which is an attribute of viral pneumonias. This was first noted with children (within September-October 2019), then with adults (within October-December, 2019). Initially we treated this phenomenon as a new form of parainfluenza. Discussions with pediatricians and general practitioners confirmed our assumptions.

Up to date, there are no clear criteria for COVID-19 treatment, while the treatment methods used before, appeared to be inefficient. We therefore chose to exercise those methods of laser therapy that could be efficient in the prevention and treatment of COVID-19. ILBI has the direct impact on all blood components, and the vascular wall. The most significant among these effects are improved microcirculation, improved rheological blood properties, as well as blood clotting reduction. For the studies of this method I received an award (Ming Chien Kao Awards 2015) [1, 2, 3]. 
For immunostimulation we used the PLT schemes, which we had used for the treatment of cancer patients, respiratory viral infections $[4,5]$. The obtained results showed that the use of combined Immunostimulation (laser + medication) could reduce the number of infected patients, as well as reduce the disease severity.

\section{Materials and Methods}

\section{Equipment for ILBI:}

The main laser therapy systems are used for ILBI:

1. Helium-neon laser -Alok-1, wavelength $632.8 \mathrm{~nm}, 1-2 \mathrm{~mW}$. (

2. Semi-conductor (diode) laser - Mulat, wavelengths $640 \mathrm{~nm} \mathrm{nm,} \mathrm{1-2}$ $\mathrm{mW}$.

3. Frequency-modulated diode laser - Magic Beam, $640 \mathrm{~nm}, 1-2 \mathrm{~mW}$.

Equipment for PLT:

1. GaAs semi-conductor laser Uzor (wavelength $890 \mathrm{~nm}$, pulsed mode, pulse power $5-10 \mathrm{~W}$.

2. Semiconductor laser Mustang (wavelength $910 \mathrm{~nm}$, pulsed mode, pulse power $10 \mathrm{~W}$.

\section{Methods of Immunostimulation:}

Stage I: ILBI (November-December, 2019). ILBI was performed according to the standard method (10 sessions) (A puncture of the ulnar vein with one-time sterile catheter was performed. The catheter consisted of a thin needle with a monofilament through which intravascular irradiation came out (produced by Polironic). After each session of irradiation, the catheter was rejected.

Stage II: Percutaneous laser therapy (Percutaneus laser therapy, hereinafter "PLT") (mid-late January, 2020). We performed percutaneous

\begin{tabular}{|l|l|l|}
\hline Age & Below 40 & $40-50$ \\
\hline 1 group & - & 1 \\
\hline 2 group & 1 & - \\
\hline 3 group & 3 & 7 \\
\hline 4 group & 2 & 2 \\
\hline
\end{tabular}

laser stimulation using the standard method during 5 days. This technique is used for Immunostimulation in cancer patients [5, 6].

Stage III: After 2 weeks, immunomodulatory drugs (Tirolone, Levamisole) were introduced according to the application schemes.

Patients of group 4: With the first signs of the disease these patients received Tilorone according to the application scheme (from 3 to 5 tablets subject to the disease severity). Vitamin C (ascorbic acid) $200 \mathrm{mg} 3$ times a day. In case the treatment appeared to be ineffective, the patients called an ambulance and were hospitalized when necessary.

\section{Results}

All patients were divided into the following groups:

Group 1: 8 patients (ILBI + PLT + immunomodulatory drugs).

Group 2: 11 patients (PLT + immunomodulatory drugs).

Group 3: 24 patients (received only medical immunostimulation).

Group 4: 8 patients (immunomodulatory drugs according to application the scheme + vitamin C). All had the signs of acute respiratory disease.

The patients were distributed by their age as follows (Table 1).

The patients were distributed by their gender as follows (Table 2).

We divided all the patients who had fallen ill according to the severity of the disease:

Mild degree: fever up to $38-39^{\circ} \mathrm{C}$, dry cough, weakness, sweating, difficulty breathing (from 1 to 3 days). Cough from 5 to 7 days. The patients had no need to be hospitalized.

Medium degree: fever up to 38-390 C, weakness, sweating, difficulty breathing (for 4-5 days). Cough up to 7-10 days. The patients had no need to be hospitalized.

\begin{tabular}{|l|l|l|}
\hline $50-60$ & $60-80$ & Over 80 \\
\hline 3 & 2 & 2 \\
\hline 4 & 4 & 2 \\
\hline 5 & 5 & 4 \\
\hline 2 & 2 & - \\
\hline
\end{tabular}

Table 1: Distribution of the patients with viral infections by age

\begin{tabular}{|l|l|l|l|l|}
\hline & 1 group & 2 group & 3 group & 4 group \\
\hline Male & 4 & 6 & 11 & 5 \\
\hline Female & 4 & 5 & 13 & 3 \\
\hline
\end{tabular}

Table 2: Distribution of patients with viral infections by gender

Severe degree: fever up to $39^{\circ} \mathrm{C}$ or higher, weakness, sweating. Pulmonary insufficiency and heart failure. Severe symptoms of intoxication. Dry barking cough. Patients needed to be hospitalized.

Two patients fell ill in Group 1. Both patients got sick during February, 2020 after their contacts with those infected with COVID-19 (one after contacts with the relatives who had come back from the UK, the other fell ill after a trip to Spain). There was detected a mild degree of the disease, the recovery came after 3 or 4 days.

Five patients fell ill in Group 2, among which 4 persons with a mild degree of the disease (2 patients in November 2019, 1 patient in December, 2019, 2 patients in the end of January-beginning of February, 2020).

Severe degree: 1 person fell ill (November, 2019).

A 17-year-old male patient fell ill abruptly. The temperature rose to $39,2^{\circ}$ C. The patient developed a severe dry cough, chills, weakness, sweating.
Immediately, Amixin was taken according to the application scheme, and vitamin C. In order to prevent secondary infections, Biseptol was taken according to the application scheme. On the next day the patient's state improved: the temperature dropped to $37,8^{\circ} \mathrm{C}$. The weakness and the sweating lessened by day 5 . The dry cough remained for 2 weeks, though. The 2nd course of PLT was performed, Amixin was re-taken according to the application scheme, as well as vitamin C. Gradually the cough lessened. The cough finally disappeared only 4 weeks after the onset of the disease. The treatment was performed at home in self-isolation. Contact persons were not identified.

Group 3: 6 persons fell ill (4 persons (end of January-beginning of February, 2020, 2 persons in mid-March, 2020).

Group 4: 8 patients (4 persons in the end of January, 2 persons in the beginning of February, 2020; 2 persons in the beginning-middle of March, 2020) were infected from relatives and acquaintances. For 2 persons the contacts were not identified. 
The results obtained for morbidity are presented in Table 3.

$1^{*}$ - (17-year-old male). The severity of the disease was assessed by the duration of dry cough and weakness which lasted for 4 weeks. The patient was not hospitalized.

$1^{* *}$ - (54-year-old male). The patient got infected at work from a COVID19 colleague. The Patient was tested for COVID-19, however the test came back negative. After 3 days the temperature increased to $39,2^{\circ} \mathrm{C}$, chills and weakness developed. There was no cough. On the 4th day in the morning the temperature dropped to $36,0^{\circ} \mathrm{C}$. The patient developed a tachycardia and a slight shortness of breath. Taking into account concomitant diseases (hypertension, obesity of the $2^{\text {nd }}$ degree, coronary heart disease, vascular atherosclerosis), the patient was hospitalized. The

\begin{tabular}{|l|l|l|l|}
\hline Severity of the disease & Mild & Medium grade & Severe degree \\
\hline Group 1 & 2 & - & - \\
\hline Group 2 & 3 & - & $1 *$ \\
\hline Group 3 & $9\left(1^{* *}\right)$ & - & - \\
\hline Group 4 & 7 & 1 & - \\
\hline
\end{tabular}

Table 3: Distribution of patients with viral infections by disease degree

\begin{tabular}{|c|c|c|c|c|c|c|c|}
\hline \multirow{2}{*}{$\begin{array}{l}\text { Groups } \\
\text { (number of } \\
\text { patients ) }\end{array}$} & \multicolumn{2}{|c|}{$\begin{array}{l}\text { A number of performed } \\
\text { tests }\end{array}$} & \multicolumn{2}{|c|}{ Results of testing } & \multirow{2}{*}{$\begin{array}{l}\text { Number of } \\
\text { sick } \\
\text { patients } \\
\end{array}$} & \multicolumn{2}{|c|}{$\begin{array}{l}\text { Reliability of the performed } \\
\text { tests, } \%\end{array}$} \\
\hline & outpatients & $\begin{array}{l}\text { in the } \\
\text { hospital }\end{array}$ & positive & negative & & outpatients & $\begin{array}{l}\text { in the } \\
\text { hospital }\end{array}$ \\
\hline $\begin{array}{l}\text { Group } 1 \\
\text { (8) }\end{array}$ & $6(2 *)$ & 2 & $3(1 *)$ & $2(1 *)$ & 2 & \multirow{4}{*}{$75,67 \%$} & \multirow{4}{*}{$95,45 \%$} \\
\hline $\begin{array}{l}\text { Group } 2 \\
\text { (11) }\end{array}$ & $7(1 *)$ & $6\left(1^{*}\right)$ & $6(1 *)$ & & 4 & & \\
\hline $\begin{array}{l}\text { Group } 3 \\
\text { (24) }\end{array}$ & $14(4 *)$ & 6 & $6(1 *)$ & $12(2 *)$ & 9 & & \\
\hline $\begin{array}{l}\text { Group } 4 \\
(8)\end{array}$ & $10(2 *)$ & 8 & 8 & $2(2 *)$ & 8 & & \\
\hline
\end{tabular}

Table 4: Results of testing patients for COVID-19

\begin{tabular}{|l|l|l|l|l|}
\hline & Number of sick patients & \multicolumn{4}{|l|}{ The distribution of patients according to the degree of the disease } \\
\hline & & mild & medium & severe \\
\hline Group 1 & $25 \%$ & $25 \%$ & - & - \\
\hline Group 2 & $36,36 \%$ & $27,27 \%$ & & $9,09 \%$ \\
\hline Group 3 & $37,5 \%$ & $37,5 \%$ & - & - \\
\hline Group 4 & $100 \%$ & $87,5 \%$ & $12,5 \%$ & \\
\hline
\end{tabular}

Table 5: Distribution of COVID-19 patients by number and severity of the disease (\%)

\section{Discussion}

For over 30 years we have been using laser energy of various wavelengths to prevent and treat various diseases $[6,7,8,9,10]$.

Near infrared radiation range $(890-910 \mathrm{~nm})$ can penetrate deeply into tissues, and have a local effect on the work of various tissues and organs. This allows you to influence the pathological focus and the work of various body systems. The use of this wavelength allows stimulating cellular immunity for up to 3 months as an average.

Using ILBI (630-640 nm) allows to directly influence the parameters of all blood cells, blood plasma, the process of clotting and the work of nerve and muscle elements of the vascular wall.

The best results with the combined use of ILBI (Group 1) can be explained by a direct impact on blood cells, improvement of microcirculation and rheological properties of blood by reducing blood clotting. Our assumptions were confirmed by the recommendations of the
Ministry of Health of Russia (there have been 6 updates of the recommendations for the treatment of COVID-19 since January, 2020). Since recently all patients in Moscow hospitals were obliged to take anticoagulants and immunomodulatory drugs.

The identical results in Groups 2 and 3 are explained by the fact that the patients' response to the introduction of immunomodulatory drugs (Group 3) is stronger than in patients receiving PLT(Group 2). The body responds to the introduction of immunomodulatory drugs with a faster production of interferons and the activation of cellular immunity. The body responds to PLT in a slower manner. As a rule, the effect comes slower, and reaches its maximum in 2 weeks. The duration of the effect is longer and lasts up to 3 months. This allows limiting the number of complications and to reduce the severity of the disease.

The scheme (PLT + immunomodulatory drugs) has been used for the treatment of acute respiratory infections for the past 5 years. As a rule, in $70-80 \%$ of cases, a positive effect was observed. The disease was stopped 
within 2 or 3 days. We have never used ILBI in these cases. Using ILBI to treat acute infections was a relative contraindication [11].

The clinical picture of COVID-19 failed to fit the picture of regular respiratory infections. It was characterized by high aggressiveness, atypical course and lack of treatment effects. The effect of frosted glass denoting the diffuse interstitial edema of the lung tissue was noted on Computed Tomography (CT) scans. All these indicated a systemic lesion of the lung tissue. ILBI's main systemic effects were reduced blood clotting and improved microcirculation. In this case, these effects could be the main ones in the complex treatment of COVID-19. In May, 2020 anticoagulants were introduced in Moscow hospitals. This proved that our choice had been correct.

In conclusion I would like to note that the presented material describes a small amount of cases, therefore the results may statistically be unreliable, while a short observation period does not provide long-term treatment results. I would just like to share the experience which was gained in the treatment of COVID-19. I trust this will help other researchers, and can support further improvements aimed at the COVID-19 treatment effectiveness.

\section{Conclusion:}

1. In the treatment of COVID-19, the use of various types of immunomodulation and anticoagulants proved to be most effective.

2. The combination of ILBI and TLT with immunomodulators proved to be the most effective in the prevention and treatment of COVID-19.

3. Immediate use of immunomodulators at the very beginning of COVID19 reduces the severity of the disease, and facilitates its course.

\section{References:}

1. Mikhailov V. (2009) Development and clinical application of Intravenous Laser Blood irradiation (ILBI). Laser Therapy. 18(2); 69- 84.

2. Mikhaylov V.A. (2015) Use of intravenous laser blood irradiation (ILBI) at 630-640 $\mathrm{nm}$ to prevent vascular diseases and to increase life expectancy. Laser Therapy, vol. 24(1); 15-26.
3. Mikhaylov V.A. (2016) Ming Chien Kao Awards 2015 Laser Therapy, mar. 25(1); 9-10.

4. Mikhailov V. A., Zakharov S. D., Skobelkin O. K., Eliseenko V. I., Chaitsev V. G., Silkina G. I., Denisov I. N. (1990) Activation of the immune system in cancer patients with low-energy laser radiation in the preoperative period. Collection of scientific and practical conference reports. Low-energy laser radiation in medical practice, Khabarovsk.

5. Mikhailov V.A., Derbenjev V.A., Denisov I.N. (1999) The use of low level laser therapy in the treatment of some pulmonary diseases (10 -years' experience). 14 th. International Congress Laser Medicine ICLM 99, Florence, 28-31 October, Suppl. Laser Journal.

6. Mikhailov V. A Skobelkin O. K. Low-energy laser in preoperative period in oncologic patients. Proceeding of the International Conference. Tashkent. 40-44.

7. Mikhailov V.A. (1998) Principles of treatment and laser therapy. Proceedings 2 Congress World Association for Laser Therapy. sept. 2-5, Kansas-Sity, USA, p.76-77.

8. Mikhailov V.A. (2000) Results of clinical study of use low level laser therapy (LLLT) for the treatment of the malignant tumors of a gastro-intestinal system. LASERS IN MEDICINE, EMLA.437455.

9. Mikhaylov V.A. A newly discovered way of the function of cardio-vascular system and the latest theory of the development of Hypertension and other cardio-vascular diseases. Adstr. " 2 nd International Conference on Hypertension \& Healthcare", Amsterdam, Netherlands, 2017, September 11-13.

10. Mikhaylov V.A. A newly discovered way of the function of cardio-vascular system and the latest theory of the development of Hypertension and other cardio-vascular diseases. EC Cardiology (ECCY), Volume 5, Issue 4 (Page No: 179-187).

11. Mikhailov V. Intravenous Laser Blood Irradiation. Greece, 2007, p.103 